\title{
Quantum Hall Ferromagnetism in Graphene
}

\author{
Kentaro Nomura ${ }^{1}$ and Allan H. MacDonald ${ }^{1}$ \\ ${ }^{1}$ Department of Physics, University of Texas at Austin, Austin TX 78712-1081, USA
}

(Dated: May 12, 2008)

\begin{abstract}
Graphene is a two-dimensional carbon material with a honeycomb lattice and Dirac-like lowenergy excitations. When Zeeman and spin-orbit interactions are neglected its Landau levels are four-fold degenerate, explaining the $4 e^{2} / h$ separation between quantized Hall conductivity values seen in recent experiments. In this paper we derive a criterion for the occurrence of interactiondriven quantum Hall effects near intermediate integer values of $e^{2} / h$ due to charge gaps in broken symmetry states.
\end{abstract}

PACS numbers: 72.10.-d,73.21.-b,73.50.Fq

Introduction - Two-dimensional graphite (graphene) is a gapless semiconductor with a honeycomb lattice and an unusual massless Dirac-fermion band structure [1] that has long attracted theoretical attention. The topology of its Bloch states leads to large momentum-space Berry phases [2], quantized and half-quantized Hall effects, and a vanishing density of states at the neutral Fermi energy which qualitatively alters the way in which electronelectron interactions 3] influence electronic properties. The integer quantum Hall effect in graphene is expected to be unusual because its Landau levels are widely separated and fourfold degenerate in the absence of weak Zeeman and spin-orbit interactions. Interest in graphene has increased recently because of experimental progress [4], including the discovery of the integer quantum Hall effect [5] with quantized values of the Hall conductivity $\left(\sigma_{x y}=4(n+1 / 2)\left(e^{2} / h\right)\right)$ separated by $4 e^{2} / h$. In this Letter we address the quantum Hall effects that should occur at intermediate integer values of filling factor $\nu$, giving rise to plateaus at $\sigma_{x y}=\nu\left(e^{2} / h\right)$, in principle for all integer values of $\nu$. These additional plateaus are expected to arise from charge gaps induced by electronelectron interactions, but have not yet been observed. They would be a new example [6] of the enhanced interaction physics that occurs at integer filling factors in a strong magnetic field whenever $N \geq 2$ Landau levels are degenerate or nearly degenerate. At integer filling factors the mean-field-theory scenario in which symmetries are broken to open gaps between quasiparticle orbitals usually applies. The ground state is then well approximated by an unrestricted Hartree-Fock state 7] in which an integer number $i<N$ of Landau levels associated with orthogonal $S U(N)$ spinors is occupied. The phenomenon of interaction induced gaps and broken symmetries at integer filling factors is known as quantum Hall ferromagnetism. The four-fold degeneracy of graphene's Landau levels follows from approximate spin-degeneracy and from Bloch state degeneracy between two inequivalent points in the honeycomb lattice Brillouin zone. The low-energy physics of graphene is well described[3] in a four-component spinor envelope-function formalism with $S U(4)$ invariant electron-electron interactions. Graphene is therefore a good example of $S U(4)$ quantum Hall ferromagnetism [8], much more accurately approximating this symmetry than bilayer electron systems $[9$ for example. The absence of additional integer Hall plateaus due to quantum Hall ferromagnetism in all but the most recent samples is almost certainly due to disorder. Fig. 1 summarizes the estimate of the minimum sample mobility required to see quantum Hall ferromagnetism in graphene which is explained below.

Massless Dirac-Weyl quasiparticles - The $\vec{k} \cdot \vec{p}$ Hamiltonian of the graphene bands is

$$
H_{0}=v\left(p_{x}+e A_{x}\right) \tau_{z} \sigma_{x}+v\left(p_{y}+e A_{y}\right) \sigma_{y}
$$

where $\tau_{z}= \pm$ labels the two-degenerate $\left(K\right.$ and $\left.K^{\prime}\right)$ valleys, $\sigma_{\alpha}$ are Pauli matrices that act in the space of the two-atoms per unit cell, and $\vec{A}(\vec{r})$ is the vector potential. In the zero field case, the Hamiltonian (11) has linear dispersion $E= \pm v \hbar k$ for both spin states and for both $K$ and $K^{\prime}$ valleys. In a magnetic field the spectrum of $H_{0}$ consists of four-fold degenerate (including

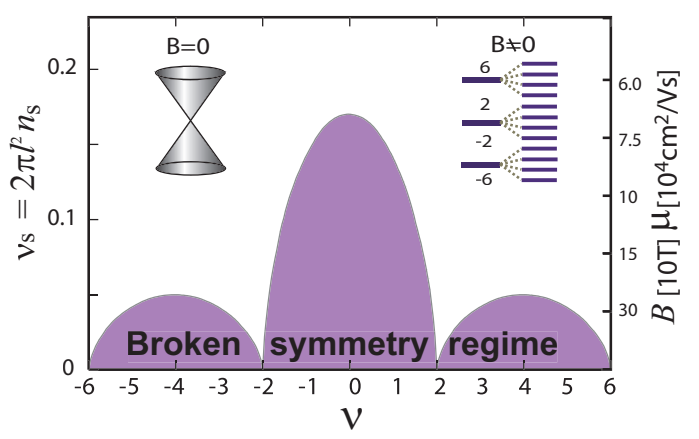

FIG. 1: Phase Diagram for $S U(4)$ quantum Hall ferromagnetism in the $n=0$ and $n=1$ Landau levels of graphene. In our model the ordered region is bounded by a maximum value of $\nu_{s}$, the ratio of the density of Coulomb scatterers to the density of a full Landau level. $\nu_{s}$ is inversely proportional to the product of the sample mobility and the external field strength and order near integer filling factors requires the minimum values for this product indicated on the right-hand vertical axis. 
spin) Landau level branches with $E_{n}= \pm \hbar v \sqrt{2 n} / \ell$ as indicated in the inset in Fig.1. For $n=0$ eigenfunctions of different valleys are localized on different honeycomb sublattices while for $n \neq 0$, they are symmetric or antisymmetric combinations of two-dimensional-electron-gas Landau level $n$ states on one sublattice and level $n+1$ states on the other sublattice. The spin degeneracy is lifted by weak Zeeman coupling that we neglect for the moment.

Stoner Criterion - The simplest approximation [10] for interacting electrons in a strong magnetic field is one in which interactions are treated in the Hartree-Fock approximation and disorder in the self-consistent Born approximation[10] (SCBA). In the strong field limit the total energy in this approximation is given by

$$
\frac{E}{N_{\phi}}=\sum_{\sigma=1}^{4}\left[\int_{-\Gamma}^{\mu_{\sigma}} d E E A(E)-\frac{X}{2} \nu_{\sigma}^{2}\right]
$$

where $N_{\phi}$ is the orbital Landau level degeneracy, $\mu_{\sigma}$ is the Fermi level for spinor-component $\sigma$,

$$
X=\int \frac{d^{2} \vec{q}}{(2 \pi)^{2}} V_{i n t}(\vec{q}) \exp \left(-q^{2} \ell^{2} / 2\right) F^{2}(\vec{q})
$$

is the exchange integral, $\ell=(\hbar c / e B)^{1 / 2}$ is the magnetic length, and $F(\vec{q})$ is a form factor we will discuss later. In the SCBA the Landau level spectral function has the form

$$
A(E)=\frac{2}{\pi \Gamma}\left[1-(E / \Gamma)^{2}\right]^{1 / 2},
$$

where $\Gamma$ is the Landau level width [10]:

$$
\frac{\Gamma^{2}}{4}=n_{s} \int \frac{d^{2} \vec{q}}{(2 \pi)^{2}}\left|U_{d i s}(\vec{q})\right|^{2} \exp \left(-q^{2} \ell^{2} / 2\right) F^{2}(\vec{q})
$$

which is estimated below. In Eqs.(3) and (5) $V_{\text {int }}(\vec{q})$ and $U_{d i s}(\vec{q})$ are the Fourier transforms of the electronelectron interaction and the disorder potential and $n_{s}$ is the density of disorder scatterers. In the case of graphene the spinor index $\sigma$ runs over four possible values. These expressions assume perfect $S U(4)$ invariance of the disorder-scattering and electron-electron interactions. While this is certainly an approximation, we believe it to be an excellent one. They also assume that spatial invariance is recovered after disorder averaging, so that the electron density matrix is diagonal in its orbital labels and the energy simply proportional to the number of orbitals in a Landau level $N_{\phi}$. In Eq.(2) the $\nu_{\sigma}$ values are the eigenvalues of the density matrix in spinor space,

$$
\nu_{\sigma}=\int_{-\Gamma}^{\mu_{\sigma}} d E A(E)
$$

which are invariant under unitary transformations of the four-dimensional spinor-space, consistent with $S U(4)$ symmetry.
In the normal state the four (nearly) degenerate Landau levels are equally occupied. To look for broken symmetry states we write $\nu_{\sigma}=\frac{\nu_{T}}{4}+\delta \nu_{\sigma}$ where $\nu_{T}=N / N_{\phi}$ is the total filling factor in the four-fold degenerate Landau level of interest. Expanding to second order in $\delta \nu_{\sigma}$ and using that $\sum_{\sigma} \delta \nu_{\sigma}=0$ we find that

$$
\begin{aligned}
\frac{E}{N_{\phi}} & =\sum_{\sigma}\left[\int_{-\Gamma}^{\mu_{0}} d E E A(E)-\frac{X}{8} \nu_{T}^{2}\right] \\
& +\sum_{\sigma} \frac{\delta \nu_{\sigma}^{2}}{2}\left[\frac{1}{A\left(\mu_{0}\right)}-X\right]+\ldots
\end{aligned}
$$

where $\mu_{0}$ is the normal state Fermi level. The normal state is unstable when the second term in square brackets is negative, in other words when $X A\left(\mu_{0}\right)>1$. This criterion for quantum Hall ferromagnetism is closely analogous to the Stoner 11] criterion for ferromagnetism in metals, and has been successfully applied[12] to understand the appearance of spin-splittings at odd integer filling factors in a semiconductor two-dimensional electron gas. In the case of quantum Hall ferromagnetism (QHF) the exchange energy competes with disorder energy rather than with band energy. We can apply the QHF Stoner criterion to graphene by relating the disorder potential to the zero-field mobility of graphene, a quantity that is conveniently available from experiment. Zero-Field Mobility and Coulomb Scattering-We start from the Boltzmann transport theory expression for the conductivity, applied to the four-fold degenerate Bloch bands of graphene:

$$
\sigma_{B=0}=\frac{e^{2} \tau v^{2} \mathcal{D}\left(E_{F}\right)}{2}=\frac{e^{2}}{h} \frac{2 E_{F} \tau}{\hbar}
$$

where $\tau$ is the scattering rate,

$$
\tau^{-1}=\frac{n_{s} k_{F}}{2 \pi \hbar^{2} v} \int_{0}^{2 \pi} d \theta\left|U_{d i s}(q)\right|^{2}(1-\cos \theta) \frac{(1+\cos \theta)}{2}
$$

$\theta$ is the scattering angle, $k_{F}$ is the Fermi wavevector and $q=2 k_{F} \sin (\theta / 2)$ is the scattering wavevector on the circular two-dimensional Fermi surface. The last $\theta$-dependent factor in Eq.( 9) is non-standard and is due to the wavevector dependence of the relative phase of graphene Bloch band wavefunctions on the two sites within its honeycomb lattice unit cell. The factor of $k_{F}$ in Eq.( 9) reflects the density dependence of the density-of-final states for elastic scattering of Fermi surface quasiparticles. For short-range scatterers the integral in Eq.( 9) remains finite as density $\propto k_{F}^{2}$ vanishes. Since $\mathcal{D}\left(E_{F}\right)$ is proportional to $k_{F}$ for two-dimensional Dirac bands, Eq. (9) implies a conductivity that is independent of $k_{F}$ and therefore independent of carrier density. Indeed theoretical studies of the conductivity of graphene 13 predict that the conductivity has a weak density dependence, remaining finite as $k_{F} \rightarrow 0$. Experiment, on the other hand, finds that the mobility 
$\mu=-\sigma / n e$ in graphene is nearly constant except at very low-densities and that it has values $\sim 10^{4} \mathrm{~cm}^{2} \mathrm{~V}^{-1} \mathrm{~s}^{-1}$ in samples that are sufficiently high quality to exhibit the integer quantum Hall effect. Evidently quasiparticle scattering amplitudes are enhanced at lower densities in such a way as to convert the $k_{F}^{+1}$ dependence of the scattering rate in Eq.(9) to a $k_{F}^{-1}$ dependence. One plausible explanation for this behavior is that Dirac band quasiparticle scattering is dominated by Coulomb scattering from charged defects near the graphene plane. For twodimensional graphene $U_{\text {dis }}(q)=V_{C}(q)=2 \pi e^{2} / q$. Inserting this expression in Eq. (9) we obtain that

$$
\frac{E_{F} \tau}{\hbar}=\frac{n}{n_{s}} \frac{4}{\pi g^{2}}
$$

where $g=\frac{e^{2}}{\hbar v} \sim 3$ is the effective fine structure constant used to characterize the ratio of Coulomb interaction and band energy scales in graphene. In Eq.(10) $n_{S}$ should be thought of as the density of Coulomb scatterers that are located in the substrate within a Fermi wavelength of the graphene plane. The influence of more remote scatterers is suppressed by the factor $\exp (-q d)$ that appears in the two-dimensional Fourier transform of the Coulomb interaction. Inserting Eq.(10) in Eq.(8) we find that mobility

$$
\mu \sim \frac{170 \mathrm{~cm}^{2} \mathrm{~V}^{-1} \mathrm{~s}^{-1}}{n_{s}\left[10^{11} \mathrm{~cm}^{-2}\right]} .
$$

In systems with Coulomb electron-electron or electronimpurity interactions screening normally plays an essential role, changing long-range interactions into shortrange ones. In a static approximation, the screened disorder potential in graphene is

$$
U_{s c}(q)=\frac{2 \pi e^{2}}{q+2 \pi e^{2} \Pi(q)}
$$

where $\Pi(q)$ is the polarization function of the graphene Dirac bands. Screening does not change the density dependence of the conductivity in graphene because $\Pi(q)$ also scales like $k_{F}$. The influence of screening on the mobility can be estimated by making a Tomas-Fermi approximation, replacing $\Pi(q)$ by $\Pi(q=0)=\mathcal{D}\left(E_{F}\right)$. When the coupling constant $g$ is much larger than 1 $U_{s c}(q) \simeq(\hbar v \pi) /\left(2 k_{F}\right)$ and

$$
\frac{E_{F} \tau}{\hbar} \simeq \frac{n}{n_{s}} \frac{64}{\pi}
$$

yielding a value for the mobility that is $16 g^{2}$ times larger than the unscreened value. We note that $g$ cancels in Eq.( 13), which is fortunate because its effective value can be influenced by non-universal substrate dielectric screening. Corrections to Eq.(13) becomes impotant for $g<1$. We can use these expressions to extract a value for the density of scatterers $n_{s}$ from measured mobilities. This procedure might retain partial validity, depending on the details, even if the limiting scatterers are not Coulombic. Similar density dependence could in principle arise from a partially accidental combination of disorder sources that gives rise to a similar increase in transition rates at small wavevectors, or from strong short-ranged scattering that approaches the unitary limit. Other potential disorder sources include random crumpling of the graphene sheet and coordination defects in the graphene sheet that give rise to long range strain fields. The procedure we now use to translate between zero-field mobilities and strong-field Landau level widths will retain its validity in some, but not all, plausible scenarios. In particular the values of $\mu B$ at the stoner phase boundary are likely to be similar for Coulomb and topological defect scatterng [14].

Self-Consistent Screening in a Magnetic Field - We are now in a position to estimate the Landau level width and apply the Stoner criterion. For Coulomb scattering

$$
\frac{\Gamma^{2}}{4}=n_{s} \int \frac{d^{2} \vec{q}}{(2 \pi)^{2}}\left|\frac{2 \pi e^{2}}{q+2 \pi e^{2} \Pi(\vec{q})}\right|^{2} \exp \left(-q^{2} \ell^{2} / 2\right) F^{2}(\vec{q}) .
$$

Notice that $\Gamma^{2}$ diverges if we neglect screening. We now need to specify the form factor $F(q)$. Taking the Coulomb interaction to be diagonal in honeycomb lattice site index it follows [10] that the form factor $F(q) \equiv 1$ for $n=0$ and that

$$
F(q)=\frac{1}{2}\left[L_{|n|}\left(q^{2} \ell^{2} / 2\right)+L_{|n|-1}\left(q^{2} \ell^{2} / 2\right)\right]
$$

for $n \neq 0$. If the magnetic field is strong enough to neglect coupling between different Landau levels the normal state polarization function $\Pi(q) \mid$ is given approximately by

$$
\Pi(q) \approx \frac{4 \exp \left(-q^{2} \ell^{2} / 2\right)}{2 \pi \ell^{2}} A\left(\mu_{0}\right) .
$$

The factor of 4 in Eq.(16) is the graphene Landau level degeneracy and the factor $\exp \left(-q^{2} \ell^{2} / 2\right)$ accounts for the orbital character of Landau level wavefunctions. Since $A\left(\mu_{0}\right)$ is proportional to $\Gamma^{-1}$, Eq. ( 14) must be solved self-consistently 15] giving rise to the following implicit equation for $\tilde{\Gamma} \equiv \Gamma /\left(e^{2} / \ell\right)$ :

$$
1=4 \nu_{s} \int_{0}^{\infty} d x \frac{F^{2} \exp \left(-x^{2} / 2\right)}{\left[\tilde{\Gamma} x+F^{2} 4 \tilde{A}_{0} \exp \left(-x^{2} / 2\right)\right]^{2}} .
$$

In Eq.( 17) $\tilde{A}_{0} \equiv \Gamma A\left(\mu_{0}\right)$ and $\nu_{s}=2 \pi \ell^{2} n_{s}$ is the 'filling factor' of scatterers. Note that since the right hand side of Eq.(17) is a monotonically increasing function of $\nu_{s}$ and a monotonically decreasing function of $\tilde{\Gamma}, \tilde{\Gamma}$ must increase monotonically with $\nu_{s}$.

Graphene QHF Phase Boundary - The Stoner criterion can be written in terms of $\tilde{\Gamma}, \tilde{A}_{0}$, and the dimensionless exchange integral

$$
\tilde{X} \equiv \frac{X}{e^{2} / \ell}=\int_{0}^{\infty} d x \frac{\tilde{\Gamma} x F^{2} \exp \left(-x^{2} / 2\right)}{\tilde{\Gamma} x+4 \tilde{A}_{0} F^{2} \exp \left(-x^{2} / 2\right)}
$$


In the absence of screening (large $\tilde{\Gamma}$ ), $\tilde{X}=\sqrt{\pi / 2}$ for $n=0$ and $\tilde{X}=(11 / 16) \sqrt{\pi / 2}$ for $n=1$. The Stoner criterion,

$$
\tilde{X} \tilde{A}_{0} / \tilde{\Gamma}>1
$$

is satisfied for $\nu_{s}>\nu_{s}^{*}$. Since $\nu_{s} \propto n_{s} / B \propto 1 / \mu B$, our Stoner criterion specifies the minimum values for the product of field and mobility illustrated in Fig. 1:

$$
B[10 \text { Tesla }] \mu\left[10^{4} \mathrm{~cm}^{2} \mathrm{~V}^{-1} \mathrm{~s}^{-1}\right] \gtrsim 1 / \nu_{s}^{*}\left(\nu_{T}\right) .
$$

The origin of the weaker tendency to ordered states in the four-fold degenerate $n=1$ Landau level is the difference in form factor $F(q)$.

Discussion - Although the Stoner criterion can be applied at all filling factors and provides a reasonable assessment of the crossover between interaction dominated and disorder dominated physics, we caution that the simple quantum Hall ferromagnet states can occur only at integer values of the total filling factor. We expect the emergence of interaction-driven gaps at intermediate integer filling factors to be the first signal that sample quality is adequate to see interaction dominated physics. Judging by the relative size of charge gaps at integer and fractional filling factors, we expect that the first fractional filling factors will require mobilities approximately five times larger than those required to realize quantum Hall ferromagnetism; the $S U(4)$ nature of these Landau levels will open up a new frontier for the fractional quantum Hall effect that is likely to yield some surprises. We have so far neglected the Zeeman energy because it is much weaker than the competing disorder and interaction energy scales and will have little influence on whether or not quantum Hall ferromagnetism occurs. (The Zeeman energy is $\sim 1 \mathrm{meV}$ at $\sim 10$ Tesla compared to a $n=0$ Landau level interaction energy scale $\sim 100 \mathrm{meV}$ depending on the degree of substrate dielectric screening.) When quantum Hall ferromagnetism does occur, however, the Zeeman energy will play a larger role. For $\nu= \pm 1$, in particular, the Zeeman energy will select ordered states that are spin-polarized, and break symmetry in the $S U(2)$ valley space. The interaction terms in graphene should be weakly dependent on valley index, because interactions on the same graphene sublattice should be more strongly repulsive than interactions between sublattices at short distances, reducing the broken symmetry to $U(1)$. For this reason, we anticipate that the $\nu= \pm 1$ quantum Hall ferromagnet in graphene should have a Kosterlitz-Thouless phase transition at a low temperature. Finally we compare our result with the recent experiment [6] that has reported quantum Hall ferromagnetism in graphene. The mobility of the sample used in [6] is $\mu=5 \times 10^{4}\left[\mathrm{~cm}^{2} / \mathrm{Vs}\right.$ ]. Fig.[ [1] indicates that for this mobility and $\nu= \pm 1$ the symmetry breaks at $17 \mathrm{~T}$ in agreement with experiment 6 . The appearance of quantum Hall plateaus observed at $\nu= \pm 4$, in the middle of the four-fold degenerate $n= \pm 1$ Landau levels at around 30Tesla is also in reasonable agreement with Fig.[1] giving the critical field 40T. The influence of dielectric screening on our phase diagram, which we expect to be rather weak, and of screening due to virtual inter-Landau-level transitions, which we expect to be important at larger $n$, will be discussed in subsequent work.

Acknowledgments-The authors acknowledge helpful interactions with Luis Brey, Andre Geim, Jason Hill, Zhigang Jiang, Philip Kim, Antonio Neto, Nuno Peres, Nikolai Sinitsyn, and Jairo Sinova. This work has been supported by the Welch Foundation and by the Department of Energy under grant DE-FG03-02ER45958.

[1] D. P. DiVincenzo and E. J. Mele, Phys. Rev. B 29, 1685 (1984); C. L. Kane and E. J. Mele, Phys. Rev. Lett. 78, 1932 (1997).

[2] G.W. Semenoff, Phys. Rev. Lett. 53, 2449 (1984); F.D.M. Haldane, Phys. Rev. Lett. 61, 2015 (1988); C.L. Kane and E.J. Mele, Phys. Rev. Lett. 95, 146802 (2005); C.L. Kane and E.J. Mele, Phys. Rev. Lett. 95, 226801 (2005); N. M. R. Peres, F. Guinea, A. H. Castro Neto, Phys. Rev. B 73, 125411 (2006). V.P. Gusynin and S.G. Sharapov, Phys. Rev. Lett. 95, 146801 (2005); N.A. Sinitsyn et al., cond-mat/0602598

[3] C. de C. Chamon et al., Phys. Rev. Lett. 77, 4194 (1996); D.H. Kim et al., Phys. Rev. Lett. 79, 2109 (1997); Y. Hatsugai and P. A. Lee, Phys. Rev. B 48, 4204 (1993); D. V. Khveshchenko, Phys. Rev. Lett. 87, 206401 (2001); J. Gonzalez et al., Phys. Rev. B 63, 134421 (2001); T. Stauber et al., Phys. Rev. B 71, 041406 (2005).

[4] Novoselov, K. S. et al., Science 306, 666 (2004); Y. Zhang et al., Phys. Rev. Lett. 94, 176803 (2005); C. Berger et al., J. Phys. Chem B 108, 19912 (2004).

[5] K.S. Novoselov et al., Nature 438, 197 (2005); Y.B. Zhang et al., Nature 438, 201 (2005).

[6] As this text was being written we learned that the anticipated integer quantum Hall effects have been discovered; Y. Zhang et al., cond-mat/0602649.

[7] Tomas Jungwirth and A.H. MacDonald, Phys. Rev. B 63, 035305 (2001).

[8] D.P. Arovas et al., Phys. Rev. B 59, 13147 (1999); Z.F. Ezawa and K. Hasebe, Phys. Rev. B 65, 075311 (2002).

[9] A.A. Burkov, A.H. MacDonald, Phys. Rev. B 66, 115320 (2002).

[10] T. Ando and Y. Uemura, J. Phys. Soc. Jpn. 37, 1044 (1974); T. Ando et al., Rev. Mod. Phys. 54, 437 (1982); A.H. MacDonald et al., Phys. Rev. B 34, 2681 (1986).

[11] E. Stoner, Proc. R. Soc. London, Ser. A 165, 372 (1938).

[12] M. M. Fogler and B. I. Shklovskii, Phys. Rev. B 52, 17366 (1995); B.A. Piot et al., Phys. Rev. B 72, 245325 (2005).

[13] E. Fradkin, Phys. Rev. B 33, 3263 (1986); P.A. Lee, Phys. Rev. Lett. 71, 1887 (1993); K. Ziegler, Phys. Rev. Lett. 80, 3113 (1998); Y. Zheng and T. Ando, Phys. Rev. B 65, 245420 (2002).

[14] S.V. Morozov, K.S. Novoselov, M.I. Katsnelson, F. Schedin, D. Jiang, A.K. Geim, cond-mat/0603826 A. 
F. Morpurgo, F. Guinea, cond-mat/0603789, J. Gonzalez, F. Guinea, M. A. H. Vozmediano, Phys. Rev. B, 63, 134421 (2001).
[15] S. Das Sarma, Phys. Rev. B 23, 4592 (1981); Y. Murayama and T. Ando, Phys. Rev. B 35, 2252 (1987). 